\title{
Tulane
}

Tulane Economics Working Paper Series

\section{Measuring Directional Mobility: The Bartholomew and Prais-Bibby Indices Reconsidered}

\author{
Satya R. Chakravarty \\ Economic Research Unit \\ Indian Statistical Institute \\ satya@isical.ac.in
}

\author{
Nachiketa Chattopadhyay \\ Statistics and Mathematics \\ Division \\ Indian Statistical Institute \\ nachiketa@isical.ac.in \\ Rodrigo Aranda Balcazar \\ Department of Economics \\ Tulane University \\ raranda@tulane.edu
}

\author{
Nora Lustig \\ Department of Economics \\ Tulane University \\ nlustig@tulane.edu
}

Working Paper 1720

December 2017

\begin{abstract}
This paper tries to interpret the Bartholomew (1967) index of social mobility in terms of a directional mobility index based on the one-step expected states of movement corresponding to a transition matrix. A partial ordering of monotone transition matrices is proposed using the generalized Lorenz ordering of expected states which can be related to social improvement in terms of the socio-economic attribute under consideration. We also interpret the Prais(1955)-Bibby ((1975) mobility index using a Bayesian approach, where the underlying mobility depends on the initial distribution of the states.
\end{abstract}

Keywords: social mobility, intergenerational mobility, monotonicity, Bayesian analysis JEL codes: C11, D31, D63, J62 


\title{
Measuring Directional Mobility: The Bartholomew and Prais-Bibby Indices Reconsidered
}

\author{
Satya R. Chakravarty, \\ Nachiketa Chattopadhyay, \\ Nora Lustig \\ and \\ Rodrigo Aranda ${ }^{1}$
}

\footnotetext{
${ }^{1}$ Satya R Chakravarty (satya@isical.ac.in, satyarchakravarty@gmail.com) works at the Economic Research Unit of the Indian Statistical Institute; Nachiketa Chattopadhyay (nachiketa@isical.ac.in) works at the Statistics and Mathematics Division of the Indian Statistical Institute; Nora Lustig (nlustig@tulane.edu) is Samuel Z. Stone Professor of Latin American Economics and Director of the Commitment to Equity Institute (CEQ), Tulane University and nonresident senior fellow of the Center for Global Development and the Inter-American Dialogue, and non-resident senior research fellow at UNUWIDER; Rodrigo Aranda (raranda@tulane.edu) is a Ph.D candidate from Tulane University Department of Economics. All errors and omissions remain the authors sole responsibility.
} 


\begin{abstract}
This paper tries to interpret the Bartholomew (1967) index of social mobility in terms of a directional mobility index based on the one-step expected states of movement corresponding to a transition matrix. A partial ordering of monotone transition matrices is proposed using the generalized Lorenz ordering of expected states which can be related to social improvement in terms of the socio-economic attribute under consideration. We also interpret the Prais(1955)-Bibby ((1975) mobility index using a Bayesian approach, where the underlying mobility depends on the initial distribution of the states.
\end{abstract}

Keywords: social mobility, intergenerational mobility, monotonicity, Bayesian analysis JEL codes: C11, D31, D63, J62 


\section{Introduction}

Measurement of socio-economic mobility has been recognized as a challenging problem in the recent past. While the theory of inequality measurement concerns itself with comparing two static income distributions at different time / regional points, it is observed that the welfare/inequality levels associated with the income distributions at certain time/regional points very well depend on the mobility of the populations from an initial state (see, among others, Shorrocks, 1978; Markandya, 1984; Kanbur and Stiglitz, 1986; Chakravarty, Dutta, and Weymark ,1985; Maasoumi and Zandvakilli,1986; Dardanoni, 1993 , Fields, 2010).

Fields and Ok( 1996, 1999) suggested a distance-based approach to the measurement of mobility. A distance-based mobility index is a summary measure of how separate are the initial and final incomes etc. Fields and Ok (1996) proposed a simple index where distance is measured by the sum of the absolute values of the difference between each person's initial and final income. A more general distance-based mobility index that relies on a ' generalized distance' of individual income differences between initial and final periods was suggested by Mitra and Ok (1998).

D’Agostino and Dardanoni (2009) studied social mobility in a framework in which social statuses of individuals are determined by their ranks. They investigated ranks of son's and father's incomes in a population and obtained a partial ordering based on what they called 'partial permutation matrices' which agrees with the standard concordance ordering of Spearman. Rank-based mobility indices were earlier considered by King (1983) and Chakravarty (1984) where higher changes in ranks of individuals between two periods were preferred to lower changes. Demuynck and Van de gaer (2010) obtained a rank dependent mobility measure based on individual mobilities defined as the ratio of final to initial period incomes, using the framework of Donaldson and Weymark (1980) and Bossert's (1990) characterization of the S-Gini index.

Mobility-as-independence measures investigate the relationship between initial and final states. Examples are correlation coefficient between initial and final incomes and 
intergenerational elasticity of income, where the latter is calculated by regressing the logarithm of parent incomes on the logarithm of child incomes. In fact, the latter formula can be expressed as the product of the correlation coefficient and the ratio between the standard deviation of the logarithm of parent incomes and that of the parent incomes ( see Black and Devereux, 2011; Foster and Rothbaum, 2012). Yitzhaki and Wodon(2004) constructed a mobility index using two-period income panel data. Their index is basically the correlation of change in income with change in ranks. As such, the minimum mobility occurs when all the incomes move in the same direction (no change in ranks) and maximum mobility arises when all the ranks are reversed.

Measurement of socio-economic mobility using transition matrices between states have been addressed in the literature (see, for example, Prais, 1955; Bartholomew, 1973; Bibby, 1975 and Shorrocks, 1978a; Markandya, 1982, 1984; Atkinson, Bourguignon and Morrison, 1992; Dardanoni, 1993, 1995; and Tsui, 2009; Van de Gaer, Schokkaert, and Martinez, 2001 and Gottschalk and Spolaore 2002). A transition matrix is a square matrix describing the individual probabilities of moving from one state to another in a dynamic system. In the literature on mobility indices based on transition matrices the class of matrices is often restricted to monotone matrices based on fractile groups. (See ,for example, Shorrocks,1978a;; Conlisk, 1989; and Dardanoni,1993). Conlisk argued that monotonicity is the most appropriate assumption in the case of Markovian model of mobility. For monotone matrices, every row $i$ first order stochastic dominates every subsequent row $i+1$ for each $i$. Hence the expected state to which movement may take place from any initial state is non-decreasing in the level of the initial or starting state. The use of fractile groups makes mobility independent of the initial group sizes of every state. Zheng (2011) used a variant of a transition matrix considering the conditional probability distributions of health status, given socioeconomic classes which are ordered. He referred to this as an income-health matrix and assumed monotonicity property to propose a measure of socioeconomic health inequality.

Dardanoni (1993) derived a social welfare function on the basis of discounted lifetime welfare prospects under different transition mechanisms. A partial ordering based on this 
welfare function is proposed as the intertemporal counterpart of Lorenz partial ordering (Dasgupta-Sen-Starrett, 1973) based on what he calls "permanent income".

In the context of ethical measurement of income mobility, Chakravarty, Dutta and Weymark (1985) also focus on welfarist measures of strongly relative income mobility, and like Shorrocks (1993), with a change being considered completely immobile if, and only if, the income shares of individuals are maintained through time.

There are many reasons for being interested in directional mobility (upward or downward) in the transition matrix-based framework. For instance, suppose the income groups in a society are ordered from the lowest to the highest. These groups can be quintiles, deciles, percentiles etc. The society may be interested in getting an overall idea about the movements of population segments from lower income groups to higher ones. This overall idea is representable by an upward income mobility index. A second example arises in the context of health mobility in a population. Consider a society with self-reported health data of the population. The six health categories 'very poor', 'poor', 'fair', 'good', 'very good' and 'excellent' are arranged in increasing order of society's preference. The movements of population proportions from lower categories of health statuses to higher categories are summarized by an upward health mobility index. Finally, an upward literacy mobility index is a summary statistic of the movements of population fractions from lower education statuses to higher ones, where education statuses are arranged from illiteracy to university education in increasing order of society's preferences.

During a period of recession or because of unemployment, downward movements of population proportions across income groups are a manifestation of downward income mobility. Worsening of health conditions of population fractions because of aging and /or sickness is an indication of downward health mobility.

In a recent contribution, Foster and Rothbaum (2012) provided a mobility framework that combines distance-based measures with the distribution-sensitivity of the transition matrix approach. Furthermore, the developed approach maintains isolation between upward and downward mobility to retain the directional knowledge. In addition to suggesting mobility 
indices, they also developed partial ordering criteria that allow unambiguous mobility inter-society and inter-temporal mobility comparisons.

One of the objectives of this paper is to interpret the Bartholomew (1967) in terms of directional mobility indices. We begin by proposing a partial ordering of monotone transition matrices using the generalized Lorenz ordering of one step expected states of movement, expected movement (for short), associated with the matrices under consideration. Since the expected states are non-decreasing in initial states, the generalized Lorenz ordering prefers the distribution with not lower cumulative expected states. Hence the ordering can be regarded as a representation of the upward mobility of two final distributions originating from an initial distribution through different transition matrices in a Markovian model. Our upward mobility ordering represents social improvement in terms of the socio-economic attribute under consideration. Since the initial distribution is assumed to be identical, the ordering and consequently the welfare of the final distribution solely depend on the transition process. Thus, the ordering is a counterpart of the Dardanoni (1993) ordering in the context of monotone mobility matrices. Taking cue from our upward ordering we consider a simple upward mobility index, which coincides with the upward sister of the well-known Bartholomew index. We also discuss a downward mobility ordering and a simple downward mobility index that becomes identical to the Bartholomew downward index. This, therefore, provides an alternative representation of the Bartholomew index.

In the literature of measuring horizontal equity, Atkinson (1980)-Plotnick(1981)Kakwani(1984) considered the change in inequality between the pre-redistribution Lorenz curve and a pre-ordered Lorenz curve of post-redistribution income, where the preredistribution rankings are fixed. Their view thus captures essentially the transition of individual incomes allowing changes in the rankings in the post redistribution with the initial ranking in the pre redistribution remaining fixed. If we look at the transition matrices between pre and post re-distribution income groups, similar analysis emerges. 
Often the states in a population may follow a particular distribution. In such a case the underlying mobility will depend on the initial distribution of the states. It is therefore logical to adopt a Bayesian probabilistic approach to measure mobility in a situation of this type. The use of equal fractile groupings helps us also in the Bayesian approach. It is shown that the Prais(1955)-Bibby ((1975) index can be rephrased as Bayesian index of mobility.

To illustrate how the Upward Mobility Index can be applied in practice, we calculated the index using data on generational mobility from the Panel Study of Income Dynamics (PSID) for the United States for individuals born between 1971 and 1986. Using data spanning from 1968 to 2013, we construct quintile transition matrices comparing the income quintile of children in 2013 with those of their parents when they were of a similar age. Since our transition matrix has 5 categories, the range for our proposed mobility index is between -2 (perfect downward mobility) and 2 (perfect upward mobility). Our results show that upward mobility for the whole population was 0.1602 . When the population is disaggregated by race into African American and White, the Upward Mobility Index was found to be equal to 0.1711 for the White population or almost 6 times higher than the 0.0308 Upward Mobility Index for the African American group. In fact, the size of the index for the African American population is indicative that movements in the upward direction are practically compensated by similar movements in the downward direction. A closer examination to the quintile transition matrices reveals that children from the White population group were more likely to experience upward mobility to the highest quintile and less downward mobility than children from the African American households group.

\section{A Mobility Ordering and the Bartholomew Indices}

Let us consider a population that has been partitioned into $k$ classes in ascending order with respect to some attribute of well-being. Examples of such well-being are income, wealth, health and so on. We write $p_{i j}$ to denote the proportion of persons belonging to class $i$ at period $t_{0}$ (initial period) moving to class $j$ at period $t_{1}$ (final period). By this 
definition, $\sum_{j=1}^{k} p_{i j}=1$ for all $i \in\{1,2, \cdots, k\}$. Consequently, $P=\left(p_{i j}\right)_{k \times k}$ is a transition matrix.

Given an initial situation, let us consider two final situations with transition matrices $P_{k \times k}$ and $P_{k \times k}^{\prime}$ respectively. For any initial state $i$, if the probability of going to any state $j$ or higher in the final situation under $P_{k \times k}$ is at least as high that under $P_{k \times k}^{\prime}$, we say that $P_{k \times k}$ does not have lower upward mobility with respect to initial state $i$. This means that starting from any initial state $i$, an individual will not have a lower chance of going to any final state $j$ or higher. Formally, the transition matrix $P_{k \times k}$ represents at least as high upward mobility as $P_{k \times k}^{\prime}$ if for all initial states $i$ and final states $j, \sum_{l=j}^{k} p_{i l} \geq \sum_{l=j}^{k} p_{i l}^{\prime}$. Equivalently, $1-\sum_{l=j}^{k} p_{i l} \leq 1-\sum_{l=j}^{k} p_{i l}^{\prime}$. That is, $\sum_{l=1}^{j-1} p_{i l} \leq \sum_{l=1}^{j-1} p_{i l}^{\prime}$ for all $j$.

This is same as the condition that every row of $P$ first order stochastic dominates every corresponding row of $P^{\prime}$. Thus, any real valued non-decreasing function of entries in any row of $P$ will be at least as large as that of any corresponding row of $P^{\prime}$. In particular, this implies that given that an individual is in an initial state $i$ will be expected to be at a final state $j$ not lower than the initial state. Thus our ordering can be restated as a vector dominance of the expected states vector, that is, the vector of states that could be arrived in the final situation from the initial situation, of $P$ over that of $P^{\prime}$.

Formally, given any two transition matrices $P_{k \times k}$ and $P_{k \times k}^{\prime}, P_{k \times k}$ is said to dominate $P_{k \times k}^{\prime}$ in terms of upward mobility if $\sum_{l=1}^{k} l p_{i l} \geq \sum_{l=1}^{k} l p_{i l}^{\prime}$ for all initial states $i=1 \ldots k$. Since this inequality has to hold for all initial states $i=1 \ldots k$, the comparison of the final situations are made under the assumption that the status quo of the initial situation is maintained (see Bourguignon,2011).This dominance criterion is quite strong in the sense that every expected final state under $P_{k \times k}$ is greater than or equal to every corresponding expected 
final state under $P_{k \times k}^{\prime}$. We can consider a weaker dominance criterion if we restrict ourselves to a special class of transition matrices.

It may be worthwhile to state that Zheng(2011) considers a similar dominance criterion for income-health monotone matrices. However, his derivation requires the monotonocity of the matrices. Our formulation, on the other hand, intuitively starts with a definition of higher (lower) directional mobility. If we now restrict ourselves to monotone matrices some results from inequality literature can be easily reformulated in the context of directional mobility.

In the literature on mobility indices based on transition matrices the class of matrices is generally restricted to monotone matrices based on fractile groups. ( See ,for example, Shorrocks,1978;Dardanoni,1993,2009 and Fields and Ok,1996)). This restriction allows better comparison and interpretation of mobility. For monotone matrices, every row $i$ first order stochastic dominates every subsequent row $i+1$ for each $i$. The use of fractile groups makes mobility independent of the initial group sizes of every state.

If we restrict the transition matrices to be monotone, the expected states vector for any transition matrix will have components in non-decreasing order. To see this formally, let $M$ denote the set of all $k \times k$ monotone transition matrices. Now, for any $P, P^{\prime} \in M$ if we denote $\sum_{l=1}^{k} l p_{i l}$ by $x_{i}$ and $\sum_{l=1}^{k} l p_{i l}^{\prime}$ by $y_{i}$, then the vectors $x=\left(x_{1}, x_{2}, \cdots, x_{k}\right)$ and $y=\left(y_{1}, y_{2}, \cdots, y_{k}\right)$ are non-decreasingly ordered.

For any $P, P^{\prime} \in M, P$ represents at least as high upward mobility as $P^{\prime}$ if $\sum_{i=1}^{m} \sum_{l=1}^{k} l p_{i l} \geq \sum_{i=1}^{m} \sum_{l=1}^{k} l p_{i l}^{\prime}$ for all $m=1 \ldots k$. This means that $x$ generalized Lorenz dominates $y$. In order to state intuitively reasonable conditions that are equivalent to the "at least as high upward mobility as" relation, it is necessary to present some preliminaries. An $n \times n$ matrix $B$ with non-negative entries is called a bistochastic matrix of order $n$ if each of its rows and columns sums to one. A function $W: \mathfrak{R}_{+}^{k} \rightarrow \mathfrak{R}$ is called S-concave if $W(x B) \geq W(x)$ for all bistochastic matrices $B$ of order, where $\mathfrak{R}_{+}^{k}$ is the non-negative part 
of the $k$ dimensional Euclidean space $\mathfrak{R}^{k}$. An S-concave function is symmetric, that is, it remains invariant under any reordering of its arguments. A function $W: \mathfrak{R}_{+}^{k} \rightarrow \mathfrak{R}$ is called $\mathrm{S}$-convex if $-W$ is S-concave .

An upward mobility index $I$ is a real valued function defined on $M$, that is, $I: M \rightarrow \mathfrak{R}^{1}$. For any $P \in M I(P)$ indicates the extent of mobility that takes place under the transition in $P$.

The following theorem, whose proof follows from Marshall and Olkin (1979, p.10) and Shorrocks (1983), can now be stated:

Theorem 1: For any $P, P^{\prime} \in M$, the following conditions are equivalent :

(i) $\sum_{i=1}^{m} \sum_{l=1}^{k} l p_{i l} \geq \sum_{i=1}^{m} \sum_{l=1}^{k} l p_{i l}^{\prime}$ for all $m=1 \ldots k$.

$(i i) I(P) \geq I\left(P^{\prime}\right)$, where $I(P)$ is of the form $\sum_{i=1}^{k} f\left(\sum_{l=1}^{k} l p_{i l}\right)$ and $f: \mathfrak{R}_{+}^{1} \rightarrow \mathfrak{R}^{1}$ is nondecreasing and concave, that is, $\sum_{i=1}^{k} f\left(\sum_{l=1}^{k} l p_{i l}\right) \geq \sum_{i=1}^{k} f\left(\sum_{l=1}^{k} l p_{i l}^{\prime}\right)$ for all non-decreasing and concave $f: \mathfrak{R}_{+}^{1} \rightarrow \mathfrak{R}^{1}$.

$(i i i) I(P) \geq I\left(P^{\prime}\right)$, where $I(P)$ is of the form $W\left(\sum_{l=1}^{k} l p_{1 l}, \sum_{l=1}^{k} l p_{2 l}, \cdots, \sum_{l=1}^{k} l p_{k l}\right)$ and $W: \mathfrak{R}_{+}^{k} \rightarrow \mathfrak{R}^{1}$ is non-decreasing and S-concave, that is, $W\left(\sum_{l=1}^{k} l p_{1 l}, \sum_{l=1}^{k} l p_{2 l}, \cdots, \sum_{l=1}^{k} l p_{k l}\right)$ $\geq W\left(\sum_{l=1}^{k} l p_{1 l}^{\prime}, \sum_{l=1}^{k} l p_{2 l}^{\prime}, \cdots, \sum_{l=1}^{k} l p_{k l}^{\prime}\right) \quad$ for all non-decreasing and S- concave functions $W: \mathfrak{R}_{+}^{k} \rightarrow \mathfrak{R}^{1}$

As stated earlier, condition (i) says that $P$ does not have lower upward mobility than $P^{\prime}$. This condition is equivalent to two other seemingly unrelated stipulations ( (ii)-(iii)) of the theorem. According to condition (ii), sum of identical non-decreasing, concave transformations of expected states of movement under $P$ is at least as high as that under 
$P^{\prime}$. Since ranking by this sum is equivalent to upward mobility ordering, we can interpret the sum $\sum_{i=1}^{k} f\left(\sum_{l=1}^{k} l p_{i l}\right)$ as an upward mobility index. Non-decreasingness of $f$ requires that the mobility index does not decrease with an increase in the position of the state expected in the terminal period. Concavity simply means that the rate of change resulting from the positional increase is non-decreasing. Therefore, in the aggregation $\sum_{i=1}^{k} f\left(\sum_{l=1}^{k} l p_{i l}\right)$ lower expected states are getting more weights. (See alsso Dardanoni (1993), where in welfare aggregation greater weights are attached to individuals starting from lower positions.) . This is intuitively reasonable. For instance, if a distribution is already at a high level of welfare given by initial state probabilities concentrated at higher welfare states, the possibility of upward mobility becomes increasingly difficult. Condition (iii) demands that a non-decreasing, S-concave function defined on the vector of expected states does not have lower value for $P$ than that for $P^{\prime}$. Evidently, $W$ can be treated as an upward mobility index. S-concavity of $W$ also ensures the aggregation involved in $W$ assigns lower weights to higher expected states.

In view of condition $(i)$ of the theorem it is evident that we can regard an increasing transformation of $\frac{1}{k} \sum_{i=1}^{k} \sum_{l=1}^{k} l p_{i l}$ as an upward mobility index. This simple functional form of the average number of states that can be expected to be arrived at the final situation from the initial situation can also be obtained by setting $f(t)=t$ in the average value of additive form $\sum_{i=1}^{k} f\left(\sum_{l=1}^{k} l p_{i l}\right)$. The function $\frac{1}{k} \sum_{i=1}^{k} \sum_{l=1}^{k} l p_{i l}$ is S-concave as well. It takes on the value $\frac{(k+1)}{2}$ in the case of perfect immobility, that is, nobody moves from respective initial states $\left(p_{i i}=1\right.$ for all $\left.i\right)$. It achieves its maximum value $k$ when all expected states are $k$, that is, $\sum_{l=1}^{k} l p_{i l}=k$ for each $l$ and the minimum value $l$ when all the expected states are 1 , the lowest state. 
Since $\frac{1}{k} \sum_{i=1}^{k} \sum_{l=1}^{k} l p_{i l}$ is concerned with upward mobility in the since that an increase in the expected state increases its value, the 0 - normalized transformation of $\frac{1}{k} \sum_{i=1}^{k} \sum_{l=1}^{k} l p_{i l}$ for perfect immobility, defined by subtracting $\frac{(k+1)}{2}$ from $\frac{1}{k} \sum_{i=1}^{k} \sum_{l=1}^{k} l p_{i l}$, becomes a suitable index of upward mobility. Formally, our upward mobility index is defined as

$$
M_{U}(P)=\frac{1}{k} \sum_{i=1}^{k} \sum_{l=1}^{k} l p_{i l}-\frac{(k+1)}{2}
$$

The index $M_{U}(P)$ is bounded above by $\frac{(k-1)}{2}$, which is achieved when each of the expected states is $k$. As stated, it takes on the value 0 if there is perfect immobility.

It becomes worthwhile to make a systematic comparison between the simple index $M_{U}$ and the well-known Bartholomew index defined as

$$
B(P)=\frac{1}{k} \sum_{i=1}^{k} \sum_{l=1}^{k}|i-l| p_{i l}
$$

This index expresses mobility in terms of average number of states crossed from the initial period $t$ to the destination period $t+s$. Thus, $B$ takes into account the distance travelled by the movers. The definition of $B$ does not assumes that the transition matrices are monotone. This non-negative index takes on the value 0 in the case of perfect immobility. $B$ also satisfies a symmetry property in the sense that an upward movement from state $i$ to the state $l$ is treated identically as a downward movement from state $l$ to state $i$.

Since $M_{U}$ is an upward mobility index, the comparison becomes valid when $B$ is concerned with pure upward mobility only. This restriction of $B$ is denoted by $B_{U}$.In such a case $p_{i j}=0$, if $i>j$. This implies that $\sum_{j=i}^{k} p_{i j}=1$ for all $i$. Hence 


$$
\begin{gathered}
\sum_{i=1}^{k} \sum_{j=i}^{k}|i-j| p_{i j}=\sum_{i=1}^{k} \sum_{j=i}^{k}(j-i) p_{i j}=\sum_{i=1}^{k} \sum_{j=i}^{k} j p_{i j}-\sum_{i=1}^{k} \sum_{j=i}^{k} i p_{i j} \\
=\sum_{i=1}^{k} \sum_{j=i}^{k} j p_{i j}-\sum_{i=1}^{k} i \sum_{j=i}^{k} p_{i j}=\sum_{i=1}^{k} \sum_{j=i}^{k} j p_{i j}-\frac{k(k+1)}{2} \text {. This shows that } \\
B_{U}(P)=M_{U}(P) .
\end{gathered}
$$

That is, the new upward mobility index $M_{U}$ coincides with the Bartholomew upward mobility index for all monotone transition matrices. A major difference between $B_{U}$ and $B$ is that the symmetry property of the latter is not relevant to $B_{U}$. Since the new index is derived using the ordering presented in Theorem 1, its exact equality with $B_{U}$ provides a new understanding of the Bartholomew (upward) mobility index. In other words, $B_{U}$ can be directly related to the ordering stated in the theorem.

It has been stated in the literature that a transition matrix with identical rows, that is, the probability of moving to any state is independent of the initial state, exhibits perfect mobility. Perfect mobility has sometimes been taken to indicate maximum mobility (Prais, 1955). Since we are concerned directional mobility, our notion of maximum upward mobility is where all persons have moved to the highest possible state. Therefore, all the elements below the diagonal or at the diagonal are zero. Perfect mobility conflicts with the Shorrocks' (1978) monotonicity axiom, which says that if an off diagonal element of a transition matrix increases at the expense of a diagonal element, then the new transition matrix represents higher mobility.

The above discussion on upward mobility can be easily extended to the case of downward mobility. Thus, we may say that for any initial state $i$, if the probability of going to any state $j$ or lower in the final situation under $P_{k \times k}$ is at least as high that under $P_{k \times k}^{\prime}$, we say that $P_{k \times k}$ does not have lower downward mobility with respect to initial state $i$. This 
means that starting from any initial state $i$, an individual will have a lower chance of going to any final state $j$ or higher. Formally, the transition matrix $P_{k \times k}$ represents at least as high downward mobility as $P_{k \times k}^{\prime}$ if for all initial state $i$ and final state $j, \sum_{l=j}^{k} p_{i l} \leq \sum_{l=j}^{k} p_{i l}^{\prime}$. Equivalently, $1-\sum_{l=j}^{k} p_{i l} \geq 1-\sum_{l=j}^{k} p_{i l}^{\prime}$. That is, $\sum_{l=1}^{j-1} p_{i l} \geq \sum_{l=1}^{j-1} p_{i l}^{\prime}$. Thus all the above discussion on upward mobility can be restated in terms of downward mobility by reversing the role of $P$ and $P^{\prime}$. Hence an upward mobility index can be considered as a downward mobility index by taking a monotone decreasing transform of the former and vice versa. A downward mobility index is S-convex and non-increasing in its arguments.

We can now define a downward mobility index $M_{D}$ by subtracting the average value of the expected number of states $\frac{1}{k} \sum_{i=1}^{k} \sum_{l=1}^{k} l p_{i l}$ from $\frac{(k+1)}{2}$, the value it achieves when there is no movement from respective initial states. Formally,

$$
M_{D}(P)=\frac{(k+1)}{2}-\frac{1}{k} \sum_{i=1}^{k} \sum_{l=1}^{k} l p_{i l}=-M_{U}(P) .
$$

The downward mobility index $M_{D}$ is bounded between $-\frac{(k-1)}{2}$ and $\frac{(k-1)}{2}$, where the lower bound represents the situation of perfect upward mobility (that is, all expected states are $k$ ) and upper bound is achieved in the scenario of perfect downward mobility, that is, when each expected state is 1 . In the situation of perfect immobility, when nobody moves, it takes on the value 0 .

In order to compare the Bartholomew (downward) index $B_{D}$ with our downward mobility index, we have to consider the case of pure downward mobility, that is, $p_{i j}=0$, if $i<j$ .This implies $\sum_{j=1}^{i} p_{i j}=1$ for all $i$. Hence, repeating the steps involved in the derivation of the relationship between the Bartholomew upward index and $M_{U}$, we have 


$$
\begin{gathered}
\sum_{i=1}^{k} \sum_{j=1}^{k}|i-j| p_{i j}=\sum_{i=1}^{k} \sum_{j=1}^{k}(i-j) p_{i j}=\sum_{i=1}^{k} \sum_{j=1}^{k} i p_{i j}-\sum_{i=1}^{k} \sum_{j=1}^{k} j p_{i j}=\sum_{i=1}^{k} i \sum_{j=1}^{i} p_{i j}-\sum_{i=1}^{k} \sum_{j=1}^{i} j p_{i j} \\
=\frac{k(k+1)}{2}-\sum_{i=1}^{k} \sum_{j=1}^{k} j p_{i j}=k M_{D}(P) . \text { Hence } \\
B_{D}(P)=M_{D}(P)
\end{gathered}
$$

From (5) it follows that the new downward mobility index coincides with the Bartholomew downward mobility index. Thus, the downward Bartholomew index is simply the negative of its upward sister. We note also that the symmetry property of $B$ is not compatible with $B_{D}$

In view Theorem 1, we can certainly consider alternative forms of $W$ to generate corresponding mobility indices. For instance, if $W$ uses a Gini-type aggregation, then its explicit form becomes

$$
W_{G}(P)=\frac{1}{k^{3}} \sum_{i=1}^{k}(2(n-i+1)) \sum_{l=1}^{k} l p_{i l} .
$$

Assignment of higher weights to lower expected states ensures S-concavity of $W_{G}$. This function also takes on its minimum value $\frac{(k+1)}{2}$ in the case of perfect immobility and its maximum value $k$ if all expected states are $k$. Following our earlier arguments, we can define the Gini upward mobility index as $M_{G U}(P)=W_{G}(P)-\frac{(k+1)}{2}$. Similarly, a Gini downward mobility index can also be defined. In fact, to every non-decreasing S-concave function of the type specified in condition $(i i i)$, we can generate corresponding upward and downward mobility indices. These indices will differ depending on how we aggregate expected states. Similar remarks applied to the functions specified in condition ( $i i$ )of the theorem. Given that we have been able to relate our simple indices given by (1) and (4) to the well-known Bartholomew index in our framework, we do not wish to proceed further along this line. 
In a recent paper, Bossert, Can and D'Ambrosio (2016) have considered a decomposition of mobility into upward and downward directional components for a head-count measure of rank mobility. Being a headcount measure, it however does not give weights to the extents of changes in the rankings.

\section{An empirical illustration}

In this section, we show how the Upward Mobility Index can be used by comparing its order of magnitude between the African American and White population in the United States. ${ }^{2}$ We calculated the index using data on generational mobility from the Panel Study of Income Dynamics (PSID) for the United States for individuals born between 1971 and 1986. The PSID is a nationally representative longitudinal household survey that started in 1968. The PSID surveys households income, employment, expenditure, health, education and other topics for 5,000 families (about 18,000 individuals). This survey allows us to track households over time and study the extent of mobility between different generations.

To analyze the extent of upward mobility for individuals born between 1971 and 1986, we selected households for which PSID has information both on parents and their children in 2013. This results in a sample of 6,748 individuals. Then we sorted individuals into quintiles for each year using the household's reported income per capita. We construct quintile transition matrices comparing the income quintile of children in 2013with those of their parents when they were of a similar age. ${ }^{3}$ For example, an individual born in 1980 would be 33 years old in 2013. His/her place in the distribution by quintiles is compared with the position of his/her parent (i.e., whomever is identified as the head of the household) when he/she was 33 years old (or thereabouts).

The resulting quintile transition matrix is shown in Table 1. The matrix describes the extent of mobility from one generation to the next. As can be observed, 40 percent of the children

\footnotetext{
${ }^{2}$ The classification is based on self-reported race/ethnicity.

${ }^{3}$ Individuals are asked to report the income of the previous year so income levels correspond to 2012.
} 
whose parents were on the lowest quintile when they were of the same age as them are still part of the lowest quintile in 2013 while only 7 percent of individuals whose parents were in the lowest quintile "migrated" to the top quintile in 2013. Regarding downward mobility at the other extreme, 51 percent of children whose parents were in the top quintile remained in the richest quintile; the remaining 49 percent, experienced downward mobility. A society with absolutely no mobility would have only ones in the main diagonal and zeros in every other cell in Table 1 and the Upward Mobility Index would equal zero. The Upward Mobility Index that corresponds to the transition matrix in Table 1 is equal to 0.1602 . To put this value into context, since our transition matrix has 5 categories, the range for our proposed mobility index is between -2 (perfect downward mobility) and 2 (perfect upward mobility). ${ }^{4}$

Table 1: Parent's and children's income quintiles, birth cohort 1971-1986

Children's income quintile in 2013

\begin{tabular}{ccccc}
\hline 1 & 2 & 3 & 4 & 5 \\
& & & & \\
0.37 & 0.25 & 0.16 & 0.15 & 0.08 \\
0.21 & 0.22 & 0.23 & 0.19 & 0.16 \\
0.14 & 0.18 & 0.21 & 0.26 & 0.21 \\
0.07 & 0.16 & 0.21 & 0.25 & 0.3 \\
0.05 & 0.12 & 0.17 & 0.24 & 0.42 \\
\hline
\end{tabular}

Source: authors calculations using the Panel Study of Income Dynamics (PSID) for the United States for individuals born between 1971 and 1986

To calculate $\mathrm{MU}(\mathrm{p})$ in equation 1 note that

$\frac{1}{k} \sum_{i=1}^{k} \sum_{j=1}^{k} l p_{i l}=3.1601743$. Hence,

\footnotetext{
${ }^{4}$ There were not enough observations to test the robustness of results to increasing the number of categories to deciles.
} 
$M U(p)=\frac{1}{k} \sum_{i=1}^{k} \sum_{j=1}^{k} l p_{i l}-\frac{k+1}{2}=0.16017$

Next, we proceeded to calculate the index for African American and White households. The resulting quintile transition matrices are shown in Tables 2 and 3, respectively. The Upward Mobility Index was found to be equal to 0.1711 for the White population or almost 6 times higher than the 0.0308 Upward Mobility Index for the African American group. In fact, the size of the index for the African American population is indicative that movements in the upward direction are practically compensated by similar movements in the downward direction. A closer examination to the quintile transition matrices reveals that children from the White population group were more likely to experience upward mobility to the highest quintile and less downward mobility than children from the African American households group

Table 2: Parent's and children's income quintiles, birth cohort 1971-1986 for African American population.

Children's income quintile in 2013

\begin{tabular}{ccccc}
\hline 1 & 2 & 3 & 4 & 5 \\
\hline 0.30 & 0.28 & 0.20 & 0.16 & 0.06 \\
0.22 & 0.27 & 0.26 & 0.11 & 0.14 \\
0.22 & 0.2 & 0.19 & 0.22 & 0.18 \\
0.12 & 0.14 & 0.16 & 0.24 & 0.34 \\
0.11 & 0.11 & 0.22 & 0.22 & 0.35
\end{tabular}

Source: authors calculations using the Panel Study of Income Dynamics (PSID) for the United States for individuals born between 1971 and 1986 
Table 3: Parent's and children's income quintiles, birth cohort 1971-1986 white population.

Children's income quintile in 2013

\begin{tabular}{|c|c|c|c|c|c|c|}
\hline & & 1 & 2 & 3 & 4 & 5 \\
\hline \multirow{5}{*}{$\begin{array}{l}\text { Parent's } \\
\text { income } \\
\text { quintile }\end{array}$} & 1 & 0.31 & 0.25 & 0.17 & 0.2 & 0.07 \\
\hline & 2 & 0.2 & 0.2 & 0.22 & 0.2 & 0.18 \\
\hline & 3 & 0.17 & 0.2 & 0.21 & 0.2 & 0.22 \\
\hline & 4 & 0.09 & 0.17 & 0.19 & 0.24 & 0.31 \\
\hline & 5 & 0.04 & 0.13 & 0.2 & 0.26 & 0.37 \\
\hline
\end{tabular}

Source: authors calculations using the Panel Study of Income Dynamics (PSID) for the United States for individuals born between 1971 and 1986

\section{A Bayesian Approach and the Prais-Bibby Index}

Often the states in a population may follow a particular distribution. In such a case the underlying mobility will depend on the initial distribution of the states. This section adopts a Bayesian probabilistic approach to measure mobility in a situation of this type.

Let the state distribution in the initial situation be represented by a random variable $X$. The corresponding distribution in the final distribution is denoted by $Y$. Let $p_{i j}$ stand for the probability that a person will be in state $j$ in the final period given that he is in state $i$ in the initial period by. Let us denote $\operatorname{Pr}(X=i)$ by $p_{i 0}$, where $\operatorname{Pr}$ denotes probability. Then by Bayes' theorem

$$
p_{i j}=\operatorname{Pr}(Y=j \mid X=i)=\frac{\operatorname{Pr}(Y=j, X=i)}{\operatorname{Pr}(X=i)},
$$

which gives $\operatorname{Pr}(Y=j, X=i)=p_{i j} p_{i 0}$. Hence 


$$
\operatorname{Pr}(X=i \mid Y=j)=\frac{\operatorname{Pr}(Y=j, X=i)}{\operatorname{Pr}(Y=j)}=\frac{p_{i j} p_{i 0}}{\sum_{i=1}^{k} p_{i j} p_{i 0}}
$$

The above probabilities sum up to 1 and hence we have a probability mass function.

We can decompose the distribution for each $j$ into three components as (i) probabilities with $i<j$ : the state $j$ is reached from inferior states indicating upward mobility, (ii) probabilities with $i>j$ : the state $j$ is reached from superior states indicating downward mobility and (iii) probability with $i=j: p_{i i}$ proportions remains immobile. When these probabilities are aggregated over states $i$ that are respectively less than $j$ and greater than $j$, the corresponding total upward mobility and downward mobility probabilities become $\frac{\sum_{i=1}^{j-1} p_{i j} p_{i 0}}{\sum_{i=1}^{k} p_{i j} p_{i 0}}$ and $\frac{\sum_{i=j+1}^{k} p_{i j} p_{i 0}}{\sum_{i=1}^{k} p_{i j} p_{i 0}}$ respectively. When $i=j$, the immobility probability is $\frac{p_{j j} p_{j 0}}{\sum_{i=1}^{k} p_{i j} p_{i 0}}$ when $i=j$,

But state $j \in\{1,2, \ldots, k\}$ is arbitrary. Therefore, by taking simple averages of these probabilities across all states $j$, we get the following Bayesian-type upward mobility, downward mobility and immobility indices, which we denote respectively $M_{B U} M_{B D}$ and $M_{B I}:$

$$
M_{B U}(P)=\frac{1}{k} \sum_{j=1}^{k} \frac{\sum_{i=1}^{j-1} p_{i j} p_{i 0}}{\sum_{i=1}^{k} p_{i j} p_{i 0}}, M_{B D}(P)=\sum_{j=1}^{k} \frac{\sum_{i=j+1}^{k} p_{i j} p_{i 0}}{\sum_{i=1}^{k} p_{i j} p_{i 0}}, M_{B I}(P)=\sum_{j=1}^{k} \frac{p_{j j} p_{j 0}}{\sum_{i=1}^{k} p_{i j} p_{i 0}} .
$$

For any transition matrix $P$, the sum of these indices equals 1 , that is, $M_{B U}(P)+$ $M_{B D}(P)+M_{B I}(P)=1$. This total establishes an exact relationship among three directional components of mobility; upward mobility, downward mobility and immobility. All these indices depend on the initial distribution of states in a population, values matrix, 
which are used as weights to the transition probabilities. If the initial states are equally likely so that $p_{i}^{0}=\frac{1}{k}, M_{B U} M_{B D}$ and $M_{B I}$ reduce respectively to the sum of probabilities above the diagonal, below the diagonal and along the diagonal respectively. An attractive feature of the indices presented in (9) is that their definitions do not depend on monotonicity of transition matrices; they are unambiguously defined for all possible transition matrices. That is, an advantage of the Bayesian approach is that it does not require monotonicity of transition matrices for defining indices of directional mobility.

We now wish to relate the indices presented in (9) with the Prais-Bibby index, which is defined as

$$
M_{P_{r} B}(P)=1-\frac{\text { trace of the transition matrix } P}{n} .
$$

This index is bounded between zero and one, where the lower bound is achieved in the case of perfect immobility and the upper bound is attained when all individuals move from their respective classes. Higher levels of movements away from the diagonal indicate higher degree of mobility. Unlike the Bartholomew index, this index ignores the distances travelled by the individuals. If the initial probabilities are the same, as in the case of percentile grouping, and if we define mobility as 1-immobility, that is, as 1- $\left(M_{B U}+M_{B D}\right)$, then this index coincides with the Prais-Bibby index. This therefore provides an exemplification of the Prais-Bibby index in the Bayesian framework. 


\section{References}

Atkinson, A. B. 1980. Horizontal equity and the distribution of tax burden, in Aron, H.J. and Boskins, M.J. (eds) The Economics of Taxation, 3-18, Brookings, Washington D.C. Atkinson, A. F. Bourguignon and C. Morrison, 1992. Empirical studies of earnings mobility. Harwood, Academic Publishers.

Aranda, R., Scott, J., 2015. CEQ Master Workbook for Mexico. Mimeo.

Bartholomew, 1973. Stochastic models for social processes, 2nd Edition, Wiley, London. Bibby, J. 1975. Methods of measuring mobility, Quality and Quantity, Springer, 107-136. Black S.E and P.J. Devereux, 2011 Recent developments in intergenerational mobility. Handbook of labor economics, 4(11), 1487-1541. Elsevier. doi:10.1016/S01697218(11)02414-2

Bossert, W. 1990, An axiomatization of the single-series Ginis, Journal of Economic Theory, 50, 82-92

Bossert, W., B Can and C. D'Ambrosio. 2016. A head-count measure of rank mobility and its directional decomposition. ECINEQ WP 2016-424.

Chakravarty S.R.1984. Normative indices for measuring social mobility, Economics Letters, 14, 175-180.

Chakravarty, S. R., B. Dutta and J.A Weymark .1985. Ethical indices of income mobility, Social Choice and Welfare, 2, 1-21.

Conlisk, J. 1989, Ranking mobility matrices, Economics Letters, 29, 231-235.

D’Agostino, M.and V. Dardanoni. 2009, The measurement of rank mobility, Journal of Economic Theory, 144, 1783-1803.

Dardanoni, V. 1993, Measuring social mobility, Journal of Economic Theory, 61, 372-394.

Dardanoni, V.1995 Income distribution dynamics: monotone Markov chains make light work. Social Choice and Welfare, 12(2), 181-192. Springer.

Dasgupta, P, A.K.Sen and D. Starrett, 1973, Notes on measurement of inequality, Journal of Economic Theory, 6, 180-187.

Demuynck, T. and D. Van de gaer 2010, Rank dependent relative mobility measures, Working Paper, 2010/628, Universiteit Gent. 
Donaldson, D . and J. Weymark 1980, A single-parameter generalization of the Gini indices of inequality, Journal of Economic Theory, 22, 67-86.

Fields,G.S., 2010 Does income mobility equalize longer-term incomes? New measures of an old concept. The Journal of Economic Inequality 8, 409-427.

Fields, G. S. and E. A. Ok 1996, The meaning and measurement of income mobility, Journal of Economic Theory, 71, 349-377.

Fields G.S. and E. Ok, 1999. The measurement of income mobility: an introduction to the literature. Springer Netherlands, 1999.

Foster, J and J. Rothbaum, 2012. Mobility curves: using cutoffs to measure absolute mobility. George Washington University, Mimeo.

Van de Gaer,D. E. Schokkaert, and M. Martinez ,2001 . Three meanings of intergenerational mobility. Economica, 68, 519-538.

Gottschalk, P. and E. Spolaore 2002. On the Evaluation of Economic Mobility. Review of Economic Studies, 69, 191-208.

Kakwani, N.C. 1984. On the measurement of tax progressivity and redistributive effect of taxes with application to horizontal and vertical equity, Advances in Econometrics, 3, 149169.

Kanbur S.M.R. and J.E.Stiglitz.1986. Intergenerational mobility and dynastic inequality, ERP 324, Princeton University, Princeton.

King, M.1983. An index of inequality with applications to horizontal equity and social mobility, Econometrica, 51, pp.99 - 115.

Maasoumi E.and S. Zandvakilli,1986 A class of generalized measures of mobility with applications, Economics Letters, Elsevier, 22, 97-102.

Markandya, A. 1982. Intergenerational exchange mobility and economic welfare. European Economic Review, 17(September 1980), 307-324.

Markandya, A. 1984. The Welfare Measurement of Changes in Economic Mobility. Economica, 51,457.

Marshall, A.W. and I. Olkin 1979, Inequalities: theory of majorization and its applications, New York, Academic Press.

Mitra T. and E. Ok 1998. The measurement of income mobility: A partial ordering approach. Economic Theory, 12, 77-102. 
Panel Study of Income Dynamics, public use dataset. Produced and distributed by the Institute for Social Research, University of Michigan, Ann Arbor, MI. 1970-2014

Plotnick, R.1981, A measure of horizontal inequity, Review of Economics and Statistics, 63, 283-288.

Prais, 1955. Measuring social mobility, Journal of Royal Statistical Society, Series A, 118, 56-66.

Shorrocks, A. F. 1978. The measurement of mobility, Econometrica, 46, 1013-1024.

Shorrocks, A.F. 1978a. Income inequality and income mobility. Journal of Economic Theory, 19, 376-393.

Shorrocks, A. F. 1983, Ranking income distributions, Economica, 50, 3-17.

Tsui, K.-yuen. 2009. Measurement of income mobility: a re-examination. Social Choice and Welfare, 33, 629-645.

Yitzhaki, S. and Q. Wodon 2004, Mobility, inequality and horizontal equity, Research on Economic Inequality, 12, 179-199.

Zheng, B. 2011. A new approach to measure socio-economic inequality in health, The Journal of Economic Inequality, 9, 555-577. 